\title{
Different effect of granulocyte colony-stimulating factor or bacterial infection on bone-marrow cells of cyclophosphamide-treated or irradiated mice
}

\author{
A. M. BUISMAN, ${ }^{\dagger}$ TH. L. VAN ZWET,* J. A. M. LANGERMANS, ${ }^{\S}$ M. F. GEERTSMA, ${ }^{*}$ P. J. M. LEENEN, $\dagger$ \\ $\&$ R. VAN FURTH* *Department of Infectious Diseases, University Hospital, Leiden, The Netherlands and $\dagger$ The Department of \\ Immunology, Erasmus University, Rotterdam, The Netherlands
}

\begin{abstract}
SUMMARY
In the present study, the effect of treatment with granulocyte colony-stimulating factor (G-CSF) on cellular composition of the bone marrow and the number of circulating leucocytes of granulocytopenic mice, whether or not infected with Staphylococcus aureus, was assessed. With two monoclonal antibodies, six morphologically distinct cell populations in the bone marrow could be characterised and quantitated by two-dimensional flow cytometry. Granulocytopenia was induced by cyclophosphamide or sublethal irradiation. Cyclophosphamide predominantly affected the later stages of dividing cells in the bone marrow resulting in a decrease in number of granulocytic cells, monocytic cells, lymphoid cells and myeloid blasts. G-CSF administration to cyclophosphamide-treated mice increased the number of early blasts, myeloid blasts and granulocytic cells in the bone marrow, which indicates that this growth factor stimulates the proliferation of these cells in the bone marrow. During infection in cyclophosphamide-treated mice the number of myeloid blasts increased. However, when an infection was induced in cyclophosphamide and G-CSF-treated mice, the proliferation of bone-marrow cells was not changed compared to that in noninfected similarly treated mice. Sublethal irradiation affected all bone-marrow cell populations, including the early blasts. G-CSF-treatment of irradiated mice increased only the number of myeloid blasts slightly, whereas an infection in irradiated mice, whether or not treated with G-CSF, did not affect the number of bone-marrow cells. Together, these studies demonstrated that irradiation affects the early blasts and myeloid blasts in the bone marrow more severely than treatment with cyclophosphamide. Irradiation probably depletes the bone marrow from G-CSFresponsive cells, while cyclophosphamide spared G-CSF responsive cells, thus enabling the enhanced G-CSF-mediated recovery after cyclophosphamide treatment. Only in these mice, bone marrow recovery is followed by a strong mobilisation of mature granulocytes and their band forms from the bone marrow into the circulation during a bacterial infection.
\end{abstract}

\section{INTRODUCTION}

Both radiotherapy and chemotherapy, used for treating neoplastic disorders, can suppress the haematopoietic activity of the bone marrow, that induces leucocytopenia, particularly granulocytopenia. The clinical benefit of accelerated recovery from granulocytopenia is a reduction of infectious complications, as granulocytes play an essential role in host defence against bacterial and fungal infections. ${ }^{1}$ To increase the number

Received 31 December 1998; revised 1 April 1999; accepted 1 April 1999.

Present address: $¥$ Research Laboratory for Infectious Diseases, National Institute for Human Health and Microenvironment, PO Box 1, Bilthoven, the Netherlands. §Biomedical Primate Research Centre, Rijswijk, the Netherlands.

Correspondence: Dr A. M. Buisman, Research Laboratory for Infectious Diseases, National Institute for Human Health and Microenvironment, PO Box 1, Bilthoven, The Netherlands. of granulocytes in the circulation, granulocytopenic patients are treated nowadays with recombinant granulocyte colonystimulating factor (G-CSF). ${ }^{2,3}$

Earlier we developed a mouse model, that in a certain way mimics the effect of prophylactic G-CSF treatment of granulocytopenic patients to prevent infection. Using this model, we reported recently that the effect of G-CSF treatment on the increase in the number of granulocytes in the circulation of granulocytopenic mice and on the course of bacterial infection in these mice, is dependent on the cause of granulocytopenia. ${ }^{4}$ In mice rendered granulocytopenic by cyclophosphamide, G-CSF induced an increase in the number of granulocytes in the circulation and at the site of infection, and enhanced the elimination of bacteria from the tissues. However, G-CSF treatment of sublethally irradiated mice did not affect the number of granulocytes in the circulation or the course of infection. These results demonstrated that irradiation and cyclophosphamide-treatment have a different effect on precursor cells in the bone marrow. 
The bone marrow contains cells of various lineages in a variety of stages of cell differentiation and maturation. Advances in two-dimensional flow cytometry allows characterisation and quantitation of these cell populations. ${ }^{5,6}$ With the use of two recently described monoclonal antibodies, murine bone-marrow cells can be separated into at least six morphologically distinct cell populations with a relatively high homogeneity, on the basis of the intensity of antigen expression. ${ }^{7}$ This method has shown to be a reliable and efficient tool for monitoring the composition of cell populations in the bone marrow of Listerial monocytogenes-infected mice ${ }^{8}$ and has also been used in a study of bone-marrow cells from granulocytopenic mice. ${ }^{9}$

The aim of the present study was to assess the effect of G-CSF on the various cell lineages in the bone marrow of mice which have been rendered granulocytopenic by cyclophosphamide or sublethal body irradiation and were infected with Staphylococcus aureus. In addition, the effect of G-CSF on the mobilisation of granulocytes into the circulation of infected mice has been studied.

\section{MATERIALS AND METHODS}

Animals

Specific pathogen-free female C57Bl/6 mice weighing $20 \mathrm{~g}$, purchased from IFFA Credo, Saint Germaine-sur-L'Abresle, France, were used. Sterilised food and tap water were given ad libitum.

\section{Induction of granulocytopenia}

Sublethal granulocytopenia was induced by single subcutaneous (s.c.) injection of $250 \mathrm{mg} / \mathrm{kg}$ bodyweight cyclophosphamide (Endoxan; Asta Medica B.V., Diemen, The Netherlands) or by sublethal total body irradiation. The animals, placed in plexiglass containers, received a total of $5 \mathrm{~Gy}$ from a ${ }^{137} \mathrm{Cs}$ gamma cell irradiator (Philips SL-75-5/6 MV linear accelerator, Philips Medical Systems, Best, The Netherlands) in the posterior-anterior and anterior-posterior directions. Both methods induced the same degree of granulocytopenia (sublethally) in $\mathrm{C} 57 \mathrm{Bl} / 6$ mice.

\section{Microorganisms}

Virulent serum-resistant $S$. aureus (ATCC 25923) were stored in tryptose phosphate broth (TPB) (Oxoid Ltd, Basingstoke, UK) supplemented with $10 \%$ dimethyl sulphoxide (DMSO) at $-70^{\circ}$. Samples of the frozen stock were cultured for $18 \mathrm{hr}$ at $37^{\circ}$ in TPB. Bacteria were collected by centrifugation (10 min; $900 \mathrm{~g}$ ), washed twice in phosphate-buffered saline (PBS) and suspended at appropriate bacterial concentrations in pyrogen-free saline. The virulence of the bacteria was maintained by repeated passage through mice.

\section{Experimental design}

After induction of granulocytopenia on day 0 , mice immediately received s.c. injections of $5 \mu \mathrm{g}$ purified recombinant human G-CSF (rhG-CSF) (Neupogen, Amgen B.V., Breda, The Netherlands) twice a day during 4 days. On day 4 of the experiment, the mice were infected intramuscularly (i.m.) with $5 \times 10^{6} \mathrm{~S}$. aureus into the right thigh muscle. After various time intervals blood was taken by puncture of the orbital venous sinus, the animals were killed by $\mathrm{CO}_{2}$ asphyxiation and both femurs were removed.
Quantitation of the number of circulating leucocytes

Blood samples were collected in plastic cups containing heparin. The numbers of leucocytes were counted in a Bürker haemocytometer and the number of granulocytes was determined by differential count of 200 leucocytes in two Giemsastained blood smears.

\section{Quantitation of the number of bone-marrow cells}

At different time-points before and after infection, mice were killed and both femurs were removed. The epiphyses were cut off and the marrow was collected by flushing the shafts with $2 \mathrm{ml}$ of RPMI medium (Flow Laboratories, Rockville, MD) supplemented with $100 \mathrm{U} / \mathrm{ml}$ penicillin $\mathrm{G}$ and $50 \mu \mathrm{g} / \mathrm{ml}$ streptomycin; then the cells were dispersed into a single cell suspension. The number of cells were counted in a Bürker haemocytometer using Türk's solution and the number of bone-marrow cells per mouse was calculated under the assumption that two femurs contain $11.8 \%$ of the total murine bone marrow. ${ }^{10}$ From freshly isolated bone-marrow cells, the erythrocytes were lysed by incubating the cells with lysis buffer $\left(0 \cdot 2 \mathrm{M} \mathrm{NH}_{4} \mathrm{Cl}, 0 \cdot 01 \mathrm{M} \mathrm{KHCO}_{3}\right.$ and $0 \cdot 1 \mathrm{~m}$ ethylenediamine tetraacetic acid (EDTA)) for $5 \mathrm{~min}$ at RT and washed three times with phosphate buffered saline (PBS).

\section{Antibodies and conjugates}

The purified monoclonal antibodies (mAb) used in this study were ER-MP12 (anti-CD31) ${ }^{4}$ and ER-MP20 (anti-Ly-6C). ${ }^{4}$ ER-MP12 was conjugated to biotin ( $N$-hydroxysuccinimidobiotin, Boehringer Mannheim GMBH, Mannheim, Germany) (ER-MP12-bio +) and ER-MP20 was conjugated to fluorescein isothiocyanate, isomer I (Sigma Chemical Co., St. Louis, MO) (ER-MP20-FITC). As a second stage fluorescent reagent R-phycoerythrin-conjugated streptavidin (SAv-PE) (Pharmingen, San Diego, CA) was used, which binds to ER-MP12-bio +.

\section{Flow cytometry}

For phenotyping $5 \times 10^{5}$ bone-marrow cells from each mouse were labelled with an optimal concentration of ERMP12-bio + in PBS supplemented with $1 \%$ bovine serum albumin (PBS-BSA) for $30 \mathrm{~min}$ at $4^{\circ}$, and then washed three times with PBS-BSA. Next, the cells were incubated with optimal concentrations of both ER-MP20-FITC and SAv-PE simultaneously during $30 \mathrm{~min}$ at $4^{\circ}$. After washing the cells three times with PBS-BSA, phenotypic analysis was performed with a FACScan ${ }^{(\infty}$ cytofluorimeter (Becton Dickinson, Sunnyvale, CA). To determine background fluorescence, cells were incubated with PBS-BSA or only one of the mAbs.

In each experiment, bone-marrow cells from naive mice were used to determine the fluorescence windows that distinguish six cell populations and these windows were used to gate the cell populations in the bone marrow of mice under study. The relative size of the various cell populations and the total numbers of bone-marrow cells per mouse were used to calculate the number of cells of each cell population in the bone marrow of each mouse.

Nomenclature of bone-marrow cell populations

In accordance with previous studies ${ }^{6,7}$ in naive mice, six cell populations could be defined as summarized in Table 1 . The intensity of antigen expression of ER-MP12 and ER-MP20 is 
Table 1. Bone-marrow cell populations of naive mice defined on the basis of binding of the monoclonal antibodies ER-MP12 and ER-MP20

\begin{tabular}{|c|c|c|c|}
\hline Cell population & ER-MP12 & ER-MP20 & FACS window in Fig. 1(a) \\
\hline Early blasts* & hi & - & 1 \\
\hline Myeloid blasts $\dagger$ & + & + & 2 \\
\hline Granulocytic cells: & - & med & 3 \\
\hline Monocytic cells $\S$ & - & hi & 4 \\
\hline Lymphoid cells & med & - & 5 \\
\hline Erythroid cells** & - & - & 6 \\
\hline
\end{tabular}

Expression measured by FACS-analysis, hi=high expression; med=mediate expression; $+=$ both high and mediate expression; $-=$ negative expression of ER-MP12 or ER-MP20.

*ER-MP12 $2^{\text {hi }}$ ER-MP20 $0^{-}$population consists of morphologically undifferentiated blasts and recognizable blasts of the myeloid, erythroid and lymphoid lineages.

$\dagger \mathrm{ER}^{-\mathrm{MP} 12^{+}} \mathrm{ER}^{-\mathrm{MP} 20^{+}}$population the myeloid blast population (ER-MP12 ${ }^{+}$ER$\mathrm{MP} 20^{+}$) contains primarily myeloid blasts, either morphologically undifferentiated or recognizable as such; the vast majority of these cells expresses the myeloid marker Gr-1 (unpublished data)

IER-MP12- ER-MP20 ${ }^{\text {med }}$ population is highly enriched for immature and mature granulocytic cells.

$\S$ ER-MP12- ${ }^{-}$ER-MP20 ${ }^{\text {hi }}$ population consists of mainly immature and mature monocytic cells.

IER-MP12 ${ }^{\text {med }}$ ER-MP20 ${ }^{-}$contains predominantly mature lymphoid cells and a few undifferentiated blasts.

**ER-MP12 ${ }^{-}$ER-MP20 ${ }^{-}$population consists of basophilic erythroblasts and more mature nucleated cells of the erythroid lineage.

indicated as: $\mathrm{hi}=$ high expression; med $=$ mediate expression; $+=$ both high and mediate expression, and $-=$ negative expression. The nomenclature of the bone-marrow cell populations, which are separated on the basis of the intensity of antigen expression, after staining the cells with the monoclonal antibodies ER-MP12 or ER-MP20 and measured by FACS-analysis (Table 1), are designated as follows: (1) early blasts (ER-MP12 ${ }^{\text {hi }}$ ER-MP20 ${ }^{-}$) which include of undifferentiated blasts and cells of the myeloid, erythroid and lymphoid cell-lineages; (2) more differentiated cells of the myeloid celllineage (ER-MP12 ${ }^{+}$ER-MP20 ${ }^{+}$), particularly myeloid blasts either morphologically undifferentiated or recognized as such, from which a vast majority of cells expresses the myeloid marker Gr-1 (P.J.M. Leenen, unpublished data); (3) granulocytic cells (ER-MP12 ${ }^{-}$ER-MP20 ${ }^{\text {med }}$ ) including band forms and mature granulocytes; (4) Monocytic cells (ER-MP12- ER-MP20 hi), which contains mainly monocyte precursors and mature monocytes; (5) lymphoid cells $\left(\right.$ ER-MP12 ${ }^{\text {med }}$ ER-MP20 ${ }^{-}$), which contains predominantly mature lymphocytes and a few undifferentiated blasts; and (6) erythroid cells (ER-MP12 ${ }^{-}$ER-MP20 ${ }^{-}$) consisting of erythroblasts and more mature nucleated cells of the erythroid celllineage.

\section{Bone marrow culture}

Nucleated bone-marrow cells were plated in triplicate at a concentration of $1 \times 10^{4}$ cells per well in a 96-well plate (Costar) in $100 \mu \mathrm{l}$ Methocult GF M3534 (Stemcell Technologies Inc., Vancouver, Canada), which stimulates the growth of granulocyte and macrophage colonies. After 7 days of culture at $37^{\circ}$ and $7 \cdot 5 \% \mathrm{CO}_{2}$, the cells were gently dispersed into a single cell suspension by dilution in PBS and the number of cells were counted in a Bürker haemocytometer using Türk's solution. Subsequently cell preparations were made by cytospin centrifugation and the numbers of granulocytes were determined by differential count of 200 cells in two Giemsa-stained bone-marrow cytospin preparations.

Statistical analysis

The significance of differences between the obtained data was determined by means of the Student's $t$-test. A value of $P<0.05$ was considered significantly different.

\section{RESULTS}

Changes in the relative size of the various murine bone-marrow cell populations after cyclophosphamide treatment or irradiation in combination with G-CSF treatment

In naive mice, six different cell populations can be distinguished (Fig. 1a). At day 4 after cyclophosphamide treatment, the relative size of both early and myeloid blast-cell populations was enlarged and that of the granulocytic cell population was markedly reduced (Fig. 1b) compared to that found in the bone marrow of naive mice (Fig. 1a). After having administered G-CSF to cyclophosphamide-treated mice during 4 days, the relative size of the mixed blast population was much larger, that of the granulocytic cell population was similar and that of the monocytic cells was strongly diminished in the bone marrow (Fig. 1c) compared to those in the bone marrow of cyclophosphamide-treated mice not given G-CSF (Fig. 1b).

At day 4 after irradiation, the relative size of all cell populations in the bone marrow had not changed (Fig. 1d) compared to that found in the bone marrow of naive mice (Fig. 1a). After 4 days G-CSF treatment to irradiated mice, the relative size of the mixed blast population was much larger, whereas the size of the granulocytic cell population, the monocytic cell population and that of the erythroid cell population in the bone marrow was much reduced (Fig. 1e) 


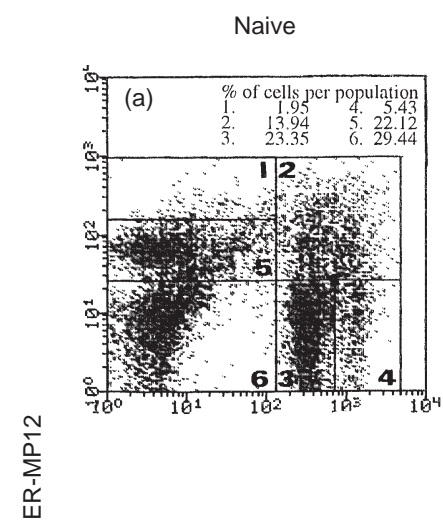

Cyclophosphamide treated
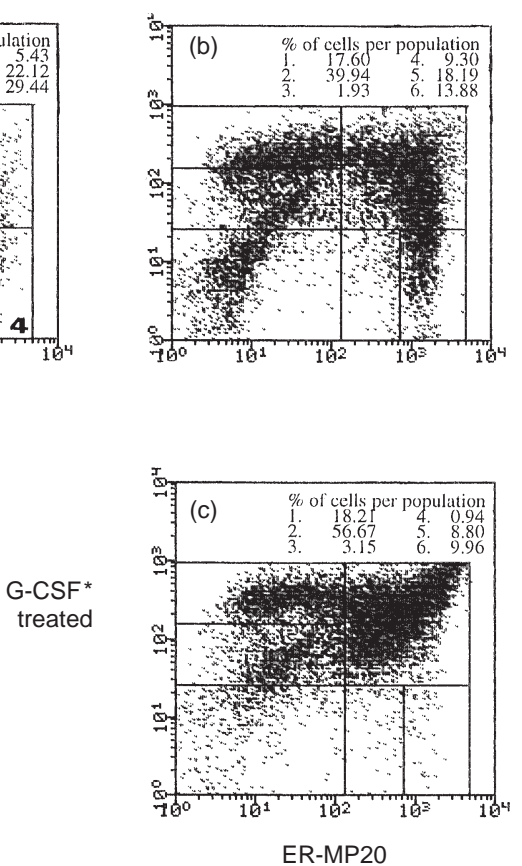

Irradiated
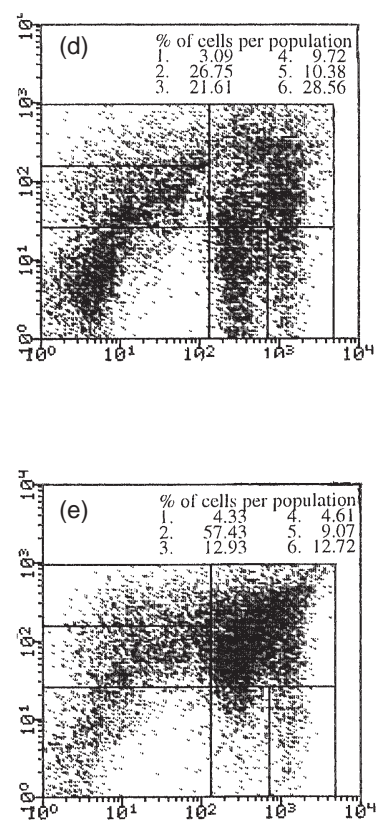

Figure 1. Two-colour flowcytrometric analysis of bone-marrow cells labeled with ER-MP12 and ER-MP20 from (a) naive mice; (b) cyclophosphamide-treated mice at day 4; (c) cyclophosphamide-treated mice after 4 days G-CSF administration; (d) irradiated mice at day 4 and (e) irradiated mice after 4 days G-CSF administration. Indicated in (a): 1. early blasts; 2 . myeloid blasts; 3. granulocytic cells; 4. monocytic cells; 5. lymphoid cells; and 6. erythroid cells. FACS-windows of one representative experiment are indicated.

compared to the respective cell populations in the bone marrow of irradiated mice not given G-CSF (Fig. 1d).

\section{Effect of G-CSF on the total number of cells in the various bone- marrow cell populations of cyclophosphamide-treated or irradiated mice}

The number of cells in each bone-marrow cell population in mice was calculated at different time-points after cyclophosphamide treatment or irradiation, whether or not combined with G-CSF-treatment. Cyclophosphamide treatment decreased the total number of cells in the bone marrow from $1.65 \times 10^{8}$ cells before treatment to $4 \cdot 4 \times 10^{7}$ cells at day 1 and to $1.5 \times 10^{7}$ cells at day 4 , i.e. a 10 -fold reduction. The number of early blasts hardly changed during 5 days of cyclophosphamide treatment (Fig. 2a). The numbers of myeloid blasts and granulocytic cells had decreased strikingly at day 1 (Fig. 2a), reaching nadir numbers at day 3 , and increased again after day 3 for the myeloid blasts and after day 4 for the granulocytic cells. The number of monocytic cells decreased also, reaching low numbers at day 2 , followed by a small increase after day 4 (Fig. 2a). The number of lymphoid cells in the bone marrow decreased from $4.8 \times 10^{7}$ cells before treatment to $1 \cdot 0 \times 10^{7}$ cells at day 1 and to $3 \times 10^{6}$ at day 4 after cyclophosphamide treatment.

After G-CSF administration to cyclophosphamide-treated mice, the number of early blasts increased slightly and the number of myeloid blasts increased strikingly $(P<0.01)$ after day 2 of G-CSF administration (Fig. 2b), whereas the numbers of the other cell populations in the bone marrow were not significantly affected (data not shown).

After irradiation, the total number of bone-marrow cells decreased from $1.9 \times 10^{8}$ cells before irradiation to $2.4 \times 10^{7}$ cells at day 1 and to $2 \cdot 5 \times 10^{7}$ cells at day 4 after irradiation, i.e. a eight-fold reduction. The total number of granulocytic cells in the bone marrow had decreased threefold and the number of the other cell populations had decreased four- to 40-fold at day 1 after irradiation (Fig. 2c). Although the number of granulocytic cells increased at day 7 and decreased again at day 11 , the numbers of the other cell populations changed hardly until day 11 after irradiation (Fig. 2c). G-CSF administration to irradiated mice increased the number of myeloid blasts in the bone marrow slightly, but did not affect the numbers of any of the various cell populations of the bone marrow (data not shown).

Effect of bacterial infection on the total number of cells of the various bone-marrow populations in cyclophosphamide-treated or irradiated mice, either treated or not treated with G-CSF

In cyclophosphamide-treated mice, the total number of bonemarrow cells did not change during the first $4 \mathrm{hr}$ of infection and increased significantly during the next $20 \mathrm{hr}$. However, a similar result was found in $24 \mathrm{hr}$ non-infected mice. G-CSFtreatment increased the total number of cells in the bone marrow of cyclophosphamide-treated mice $(P<0 \cdot 01)$; during infection in these mice only a slight increase in the total number of bone-marrow cells was found, relative to that in 


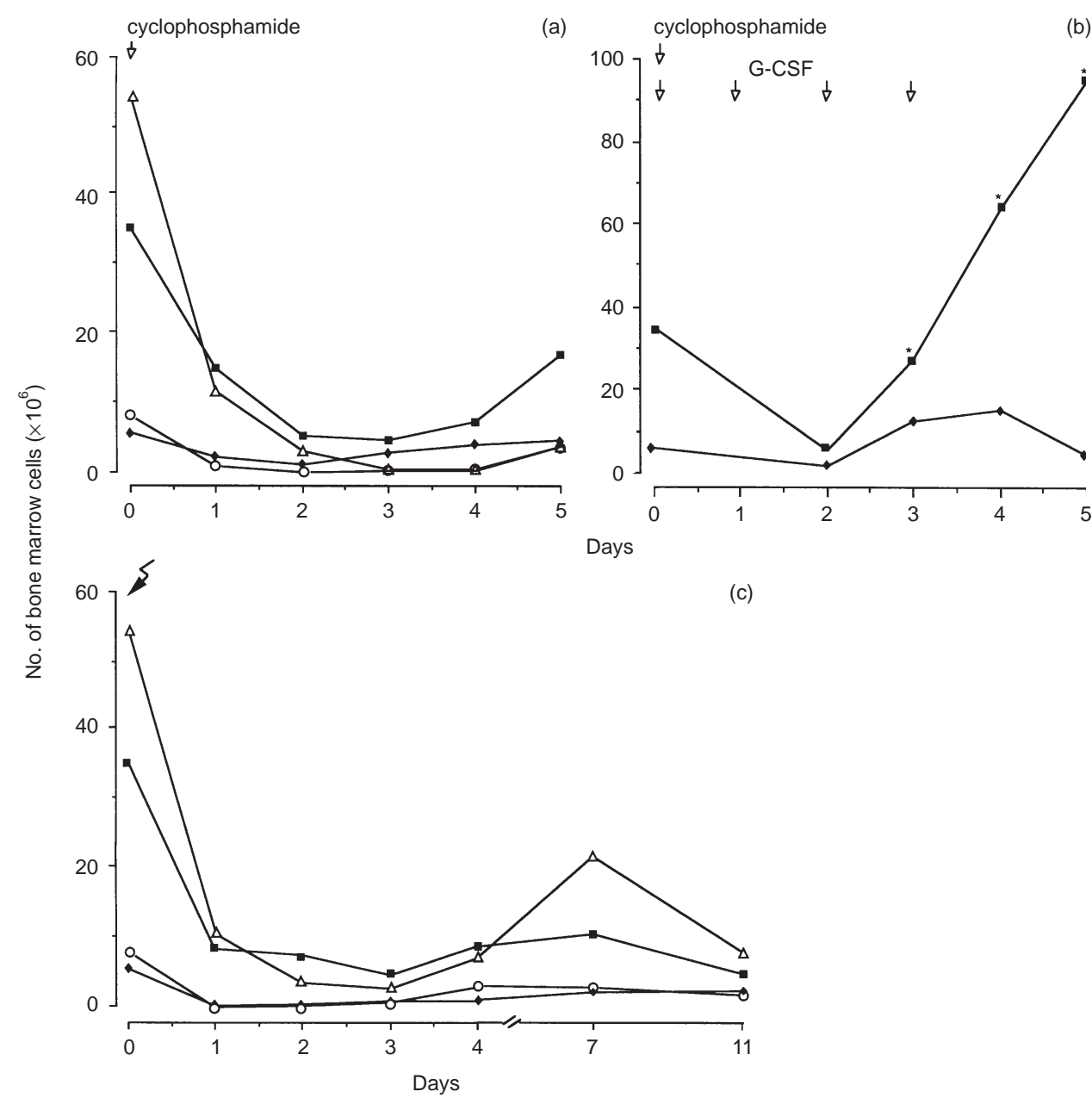

Figure 2. Number of bone-marrow cells at different time-intervals after (a) s.c. treatment with $250 \mathrm{mg} / \mathrm{kg}$ cyclophosphamide (b) after sc. treatment with cyclophosphamide followed by G-CSF administration during 4 days, and (c) after sublethal irradiation.Numbers of early blasts $(\diamond)$, myeloid blasts $(\mathbf{\square})$, granulocytic cells $(\triangle)$ and monocytic cells $(O)$ are presented. $n=4-8$ mice at each time-point. ${ }^{*}=$ significant difference comparing G-CSF treated mice with non-G-CSF-treated mice

non-infected, G-CSF-treated mice (Fig. 3a). During infection in irradiated mice, whether or not treated with G-CSF, the total number of bone-marrow cells did not change (Fig. 3a).

The total number of bone-marrow cells of cyclophosphamide-treated or irradiated mice (Fig. 3a), whether or not, treated with G-CSF and/or infected with $S$. aureus, and the relative size of the various bone-marrow cell populations (data not shown) of these mice were used to calculate the total number of early blasts (Fig. 3b), myeloid blasts (Fig. 3c), granulocytic cells (Fig. 3d) and monocytic cells (Fig. 3e) in the bone marrow.

During a $S$. aureus infection in cyclophosphamide-treated mice the number of myeloid blasts (Fig. 3c) increased and the number of monocytic cells (Fig. 3e) decreased; in these mice also the number of early blasts (Fig. 3b) and granulocytic cells (Fig. 3d) increased significantly, however, a similar result was found in $24 \mathrm{hr}$ non-infected cyclophosphamide-treated mice. G-CSF-treatment of cyclophosphamide-treated mice increased the number of early blasts (Fig. 3b), myeloid blasts (Fig. 3c) $(P<0 \cdot 01)$ and granulocytic cells (Fig. 3d), and decreased the number of monocytic cells (Fig. 3e). During infection after G-CSF administration to cyclophosphamide-treated mice, the number of early blasts decreased (Fig. 3b), whereas the number of granulocytic cells (Fig. 3d), and monocytic cells (Fig. 3e) increased, but this increase was similar to that in non-infected G-CSF treated mice.

In irradiated mice, the number of granulocytic cells (Fig. 3d) and monocytic cells (Fig. 3e) decreased during infection. G-CSF-treatment of irradiated mice increased the number of myeloid blasts $(P<0 \cdot 01)$ (Fig. 3c); however, an infection in these mice did not change the number of any of the bonemarrow cell populations.

\section{Proliferation of bone-marrow cells in culture}

To study whether the repopulating ability of the bone-marrow cells is affected by cyclophosphamide treatment, irradiation, G-CSF-treatment and/or bacterial infection, bone-marrow cells of all these mice under study were cultured in vitro. Bonemarrow cells from cyclophosphamide-treated, noninfected mice, had a significantly higher proliferative activity in culture, relative to those of naive mice (Table 2). When cyclophosphamide-treated mice were treated with G-CSF, whether or not infected with $S$. aureus, their bone-marrow cells proliferated significantly more in comparison with those from non- 
(a) Total bone-marrow cells

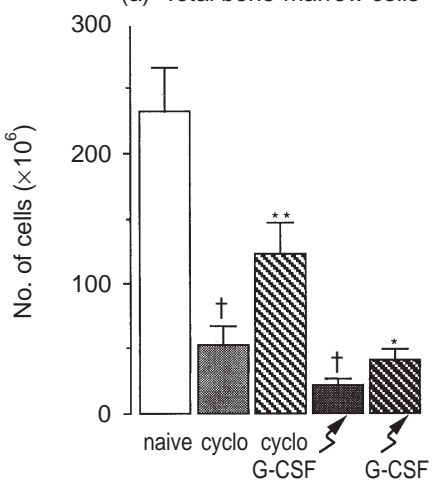

Non-infected

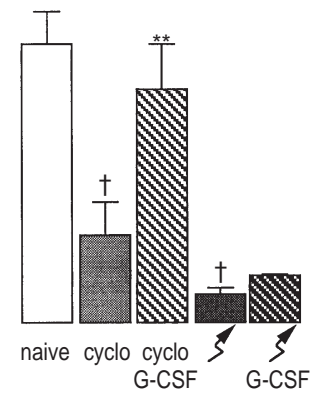

$4 \mathrm{hr}$ after infection

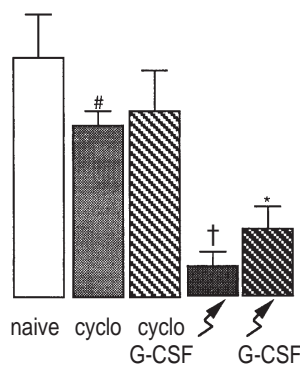

$24 \mathrm{hr}$ after infection

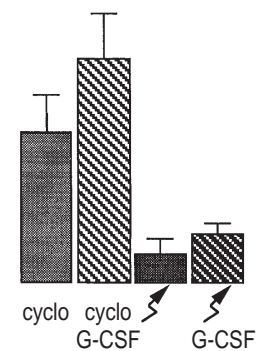

$24 \mathrm{hr}$ non-infected

(b) Early blasts

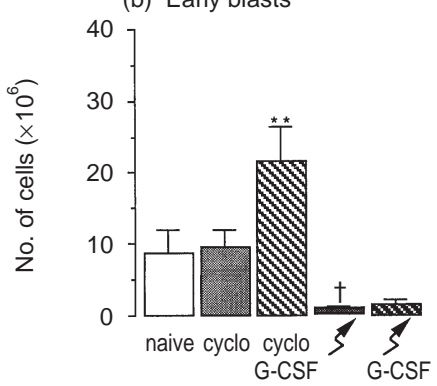

Non-infected

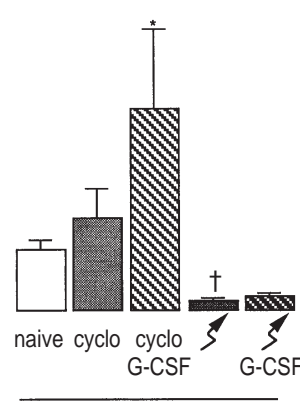

$4 \mathrm{hr}$ after infection

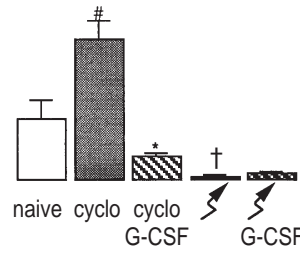

$24 \mathrm{hr}$ after infection

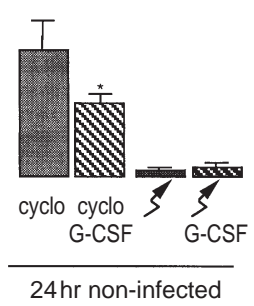

(c) Myeloid blasts
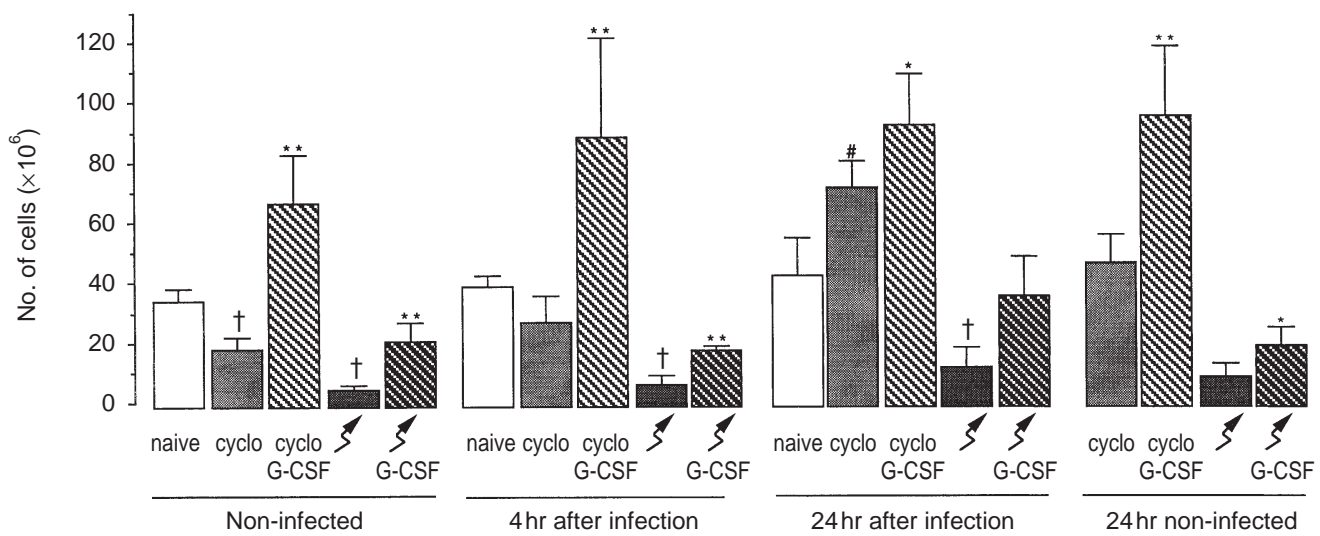

Figure 3. Number of (a) total cells; (b) early blasts; (c) myeloid blasts; (d) granulocytic cells and (e) monocytic cells in the bone marrow from naive, cyclophosphamide-treated mice or irradiated mice whether or not treated with G-CSF; before infection, at 4 and $24 \mathrm{hr}$ after infection with $S$. aureus, and $24 \mathrm{hr}$ later without infection in these mice. Mice were treated with cyclophosphamide or irradiated, thereafter treated with G-CSF or vehicle for 4 days and subsequently infected with $5 \times 10^{6}$ bacteria in the thigh muscle; control mice were not infected and studied $24 \mathrm{hr}$ later. Values are mean and sd of 4-10 mice at each time-point. ** significant difference comparing G-CSF-treated with non-G-CSF-treated $(P<0.01)$; *significant difference comparing G-CSF-treated with non-G-CSF-treated $(P<0 \cdot 05)$; †significant difference relative to naive mice $(P<0.05)$.

G-CSF, similarly treated, mice (Table 2). The proliferation of bone-marrow cells from $S$. aureus infected, cylophosphamidetreated mice, was lower than that of non-infected cyclophosphamide-treated mice independent of G-CSF treatment (Table 2).

Bone-marrow cells from irradiated mice, whether or not infected with $S$. aureus, hardly showed any proliferation during 7 days of culture (Table 2). However, G-CSF-treatment of irradiated mice resulted in a significant increased proliferation of bone-marrow cells, but, when G-CSF-treated, irradiated, mice were infected for $24 \mathrm{hr}$, their bone-marrow cells did not proliferate (Table 2).

Because the percentages of granulocytes in the bonemarrow cultures from all mice under study were about similar (Table 2), the differences in the total number of granulocytes in bone-marrow cell cultures obtained from mice under various experimental conditions showed the same variations as the total number of cells after 7 days of culture (Table 2). 


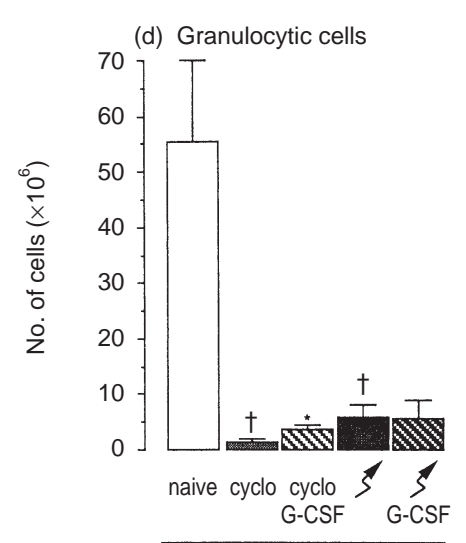

Non-infected

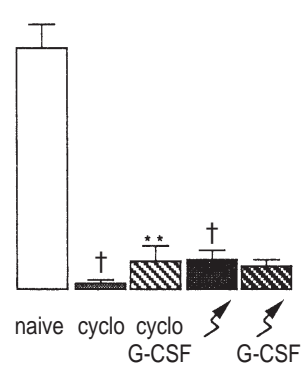

$4 \mathrm{hr}$ after infection

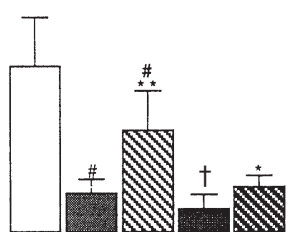

naive cyclo cyclo $\$$ G-CSF

$24 \mathrm{hr}$ after infection

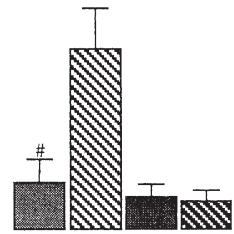

cyclo cyclo $\$$ S

$24 \mathrm{hr}$ non-infected

(e) Monocytic blasts
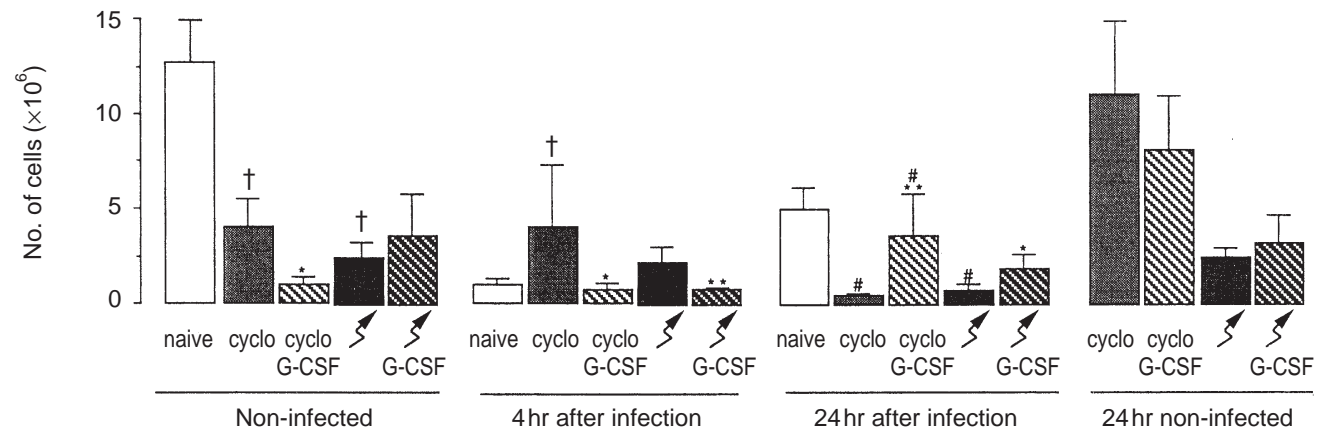

Figure 3. (Continued)

Table 2. Proliferation of bone-marrow cells from naive, cyclophosphamide-treated and irradiated mice treated with G-CSF and infected with $S$. aureus†

\begin{tabular}{|c|c|c|c|c|c|}
\hline Mice & G-CSF & $\begin{array}{l}\text { Time after } \\
\text { infection } \\
\text { (h) }\end{array}$ & $\begin{array}{l}\text { Total number of } \\
\text { cells after } 7 \text { days } \\
\qquad\left(\times 10^{4}\right)\end{array}$ & $\begin{array}{l}\text { Percentage of } \\
\text { granulocytes: }\end{array}$ & $\begin{array}{l}\text { Total number } \\
\text { of granulocytes } \\
\qquad\left(\times 10^{4}\right) \ddagger\end{array}$ \\
\hline \multirow[t]{3}{*}{ Naive } & - & 0 & $12 \cdot 5(3 \cdot 5)$ & $33 \cdot 3(10 \cdot 7)$ & $4 \cdot 9(2 \cdot 3)$ \\
\hline & - & 4 & $24 \cdot 3(24 \cdot 8)$ & $32 \cdot 5(5 \cdot 5)$ & $1 \cdot 8(1.6)$ \\
\hline & - & 24 & $15 \cdot 5(9 \cdot 9)$ & $20 \cdot 2(2 \cdot 8)$ & $3 \cdot 1(2 \cdot 0)$ \\
\hline \multirow[t]{6}{*}{ Cyclophosphamide-treated } & - & 0 & $51 \cdot 0(1 \cdot 1) \S$ & $30 \cdot 5(2 \cdot 8)$ & $16 \cdot 0(0 \cdot 8) \S$ \\
\hline & + & 0 & $110 \cdot 7(24 \cdot 5)^{* *}$ & $28 \cdot 7(10 \cdot 7)$ & $33 \cdot 1(17 \cdot 4)^{* *}$ \\
\hline & - & 4 & $15 \cdot 1(3 \cdot 5)$ & $20 \cdot 5(0 \cdot 5)$ & $3 \cdot 1(0 \cdot 7)$ \\
\hline & + & 4 & $118.0(10 \cdot 5)^{* *}$ & $23 \cdot 0(5 \cdot 1)$ & $27 \cdot 4(8 \cdot 0)^{* *}$ \\
\hline & - & 24 & $25 \cdot 4(8 \cdot 7)$ & $24 \cdot 2(6 \cdot 2)$ & $6 \cdot 7(4 \cdot 2)$ \\
\hline & + & 24 & $40 \cdot 0(10 \cdot 4)$ & $22 \cdot 5(2 \cdot 7)$ & $9 \cdot 0(2 \cdot 4)$ \\
\hline \multirow[t]{6}{*}{ Irradiated } & - & 0 & $1 \cdot 2(0 \cdot 7) \S$ & $20 \cdot 8(5 \cdot 6)$ & $0 \cdot 2(0 \cdot 1) \S$ \\
\hline & + & 0 & $7 \cdot 3(5 \cdot 2)^{*}$ & $24 \cdot 2(3 \cdot 5)$ & $1.6(0.9)^{*}$ \\
\hline & - & 4 & $1 \cdot 3(0 \cdot 7) \S$ & $22 \cdot 2(5 \cdot 7)$ & $0 \cdot 3(0 \cdot 1) \S$ \\
\hline & + & 4 & $6 \cdot 0(3 \cdot 1)^{*}$ & $25 \cdot 7(1 \cdot 1)$ & $1.5(0.8)^{*}$ \\
\hline & - & 24 & $1.8(1.9)$ & $19 \cdot 0(6 \cdot 1)$ & $0.4(0.5)$ \\
\hline & + & 24 & $1.4(0.4)$ & $27 \cdot 7(2 \cdot 1)$ & $0 \cdot 4(0 \cdot 1)$ \\
\hline
\end{tabular}

$\dagger$ Mice were treated with cyclophosphamide or irradiated, then treated with G-CSF or vehicle for 4 days and subsequently either or not infected with $5 \times 10^{6} \mathrm{~S}$. aureus in the thigh muscle. Before infection and at 4 and $24 \mathrm{hr}$ after infection, bone-marrow cells from the mice were isolated and $1 \times 10^{4}$ bone marrow were cultured for 7 days in triplicate, then the total number of cells present and the number of granulocytes were determined. values are mean (SD) of three mice at each time-point.

†After 7 days of culture granulocyte precursors are differentiated into mature granulocytes.

$\S$ Significant difference relative to naive mice $(P<0 \cdot 05)$.

**Significant difference comparing G-CSF-treated with non-G-CSF-treated $(P<0 \cdot 01)$

*Significant difference comparing G-CSF-treated with non-G-CSF-treated; $(P<0 \cdot 05)$. 
Effect of G-CSF on the number of granulocytes in peripheral blood of cyclophosphamide or irradiated mice whether or not infected with $S$. aureus.

After cyclophosphamide treatment, the number of circulating granulocytes reached nadir values at day 4 (Fig. 4), which remained unchanged at day 5 (data not shown). During infection of cyclophosphamide-treated mice, the number of blood granulocytes had not changed at $4 \mathrm{hr}$, but increased $(P<0.05)$ during the next $20 \mathrm{hr}$, relative to non-infected mice. G-CSF administration to cyclophosphamide-treated mice increased the number of circulating granulocytes $(P<0 \cdot 05)$ (Fig. 4). In cyclophosphamide-treated, infected mice, which were treated with G-CSF, the number of circulating granulocytes increased 10 -fold $(P<0 \cdot 01)$ at $24 \mathrm{hr}$ compared to noninfected, similarly treated mice (Fig. 4$)$.

In irradiated mice, the number of circulating granulocytes reached low values at 4 days and during infection the number of blood granulocytes did not change (Fig. 4). G-CSF treatment of irradiated mice did not affect the number of granulocytes in the peripheral blood (Fig. 4).

\section{DISCUSSION}

In the present study we focused on the stimulatory effects of G-CSF on various bone-marrow cell populations of granulocytopenic mice, whether or not infected with $S$. aureus. Differences were found between mice rendered granulocytopenic by cyclophosphamide treatment or by sublethal irradiation. The main conclusions that could be drawn from these studies are that cyclophosphamide treatment spares the early blast population but affects the other cell populations in the bone marrow, whereas sublethal irradiation affects all bone-marrow cell populations.

When we consider cyclophosphamide-treated, non-infected mice, G-CSF increased both the number of early and to a greater extent, the number of myeloid blasts. During an $S$. aureus infection in these mice, the number of the myeloid blasts increased further, and conceivably this leads to an increased number of granulocyte precursors in the bone marrow, which can not be identified as a separate class of cells with the monoclonal antibodies used. Ultimately this results in a large increase in the number of mature granulocytes, including band forms, in the circulation. However, in irradiated mice, whether or not infected, G-CSF increased only slightly the number of myeloid blasts and did not affect the number of circulating granulocytes. The results of the present study are in agreement with our earlier study demonstrating no effect of G-CSF treatment on the course of a $S$. aureus infection of the thigh muscle in sublethally irradiated mice, while in cyclophosphamide-treated mice, that had been administered G-CSF, the elimination of bacteria in thigh muscle, spleen and liver was significantly faster compared to non-GCSF similarly treated mice. ${ }^{4}$ The results of these in vivo studies are supported by in vitro cultures of bone-marrow cells of the mice under the various experimental conditions applied. The most striking difference was that in cultures of bone-marrow cells from cyclophosphamide-treated, $S$. aureus-infected mice, that had received G-CSF, the proliferation of granulocyte precursors resulting in mature granulocytes, was many-fold greater than in sublethally irradiated, similarly treated mice.

When we speculate about the differences in response to G-CSF treatment between cyclophosphamide-treated and irradiated mice, we may assume that in the bone marrow of cyclophosphamide-treated mice G-CSF-responsive cells are still present, while in irradiated mice these cells can not respond any more or have been eliminated. The difference in response to G-CSF between cyclophosphamide-treated and irradiated mice can not be explained by a difference in the degree of granulocytopenia; granulocytopenia was even more severe in the cyclophosphamide-treated mice compared to the irradiated mice.

Sublethal irradiation of naive mice damaged all subpopulations of the bone marrow, including the early blasts. Irradiation affects only active cycling cells during DNA-synthesis ${ }^{11}$ and the recovery after irradiation is influenced primarily by the surviving, resting stem cells. ${ }^{12-15}$ In contrast, it is reported that
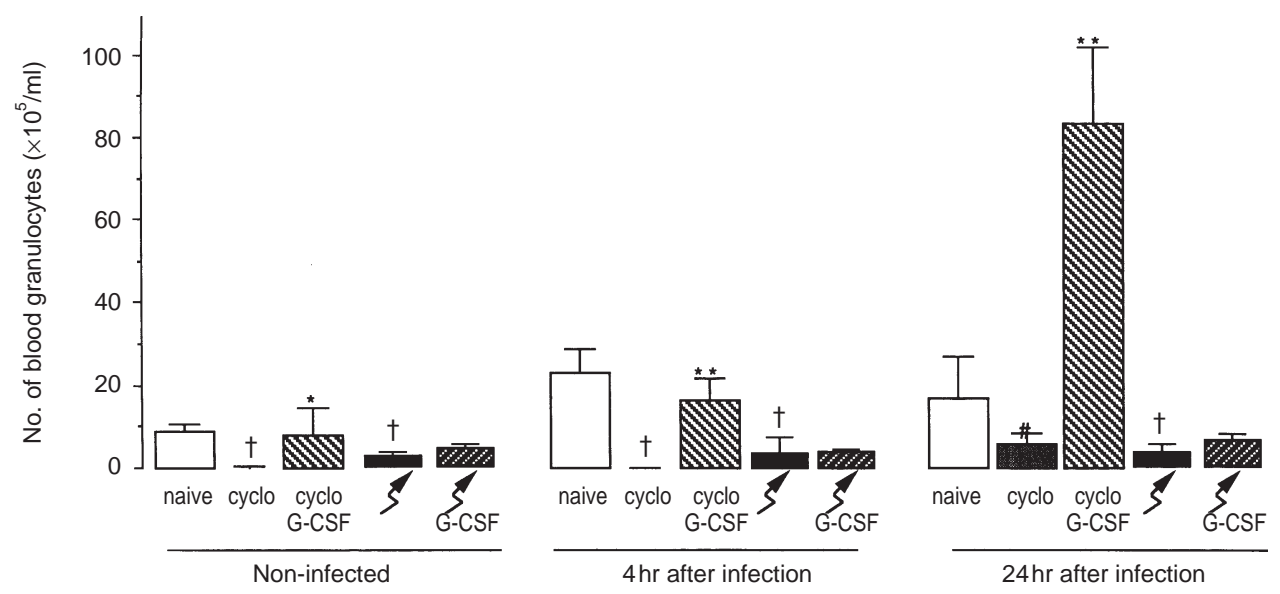

Figure 4. Number of granulocytes in peripheral blood of naive, cyclophosphamide-treated mice and irradiated mice whether or not treated with G-CSF before infection and at 4 and $24 \mathrm{hr}$ after infection with $S$. aureus in these mice. Mice were treated with cyclophosphamide or irradiated, thereafter treated with G-CSF or vehicle for 4 days and subsequently infected with $5 \times 10^{6}$ bacteria in the thigh muscle. Values are mean and sd of 4-10 mice at each time-point. **significant difference comparing G-CSF-treated with non-G-CSF-treated $(P<0 \cdot 01)$; *significant difference comparing G-CSF-treated with non-G-CSF-treated $(P<0 \cdot 05)$; $\dagger$ significant different relative to naive mice $(P<0 \cdot 05)$. 
bone-marrow cells in the S-phase of cell cycle are more radioresistant than other cells. ${ }^{16}$ In our experiments, cultures of bone-marrow cells from irradiated mice, whether or not treated with G-CSF, did not show any substantial increase, but also no decrease in cell number, which indicates that a great majority of dividing cells, probably cells not in S-phase, are affected by irradiation.

Apparently, in vivo, the delay of bone-marrow recovery exhibited by the increase in the numbers of bone-marrow cells and circulating granulocytes after G-CSF treatment differed also between irradiated and cyclophosphamide-treated mice. After irradiation and G-CSF treatment bone-marrow recovery did not occur during the first 11 days, while after cyclophosphamide and G-CSF treatment the number of bone-marrow cells increased after day 4 . This delay in bone-marrow recovery can explain the absence of an effect of G-CSF treatment on the number of circulating granulocytes after irradiation. However, others have described radioprotective effects of G-CSF on the survival of mice after lethal body irradiation, ${ }^{17-19}$ but these data were obtained from days 12-21 after irradiation and G-CSF treatment. The data in our experiments covered only day 0 to day 11 after irradiation and G-CSF administration. This indicates the importance of the duration of the G-CSF treatment after irradiation. It is conceivable that during time, stem cells which survived irradiation will become sensible for G-CSF and proliferate much faster. Our studies, however, were focussed on the role of G-CSF during the first 5 days after the granulocytopenia, as we wanted to explain the different effects of G-CSF on the course of a $S$. aureus infection found during granulocytopenia induced by irradiation or cyclophosphamide treatment, which we reported earlier. ${ }^{4}$

It is generally accepted that cyclophosphamide and other alkylating agents do not affect cells in a resting phase of the cell cycle, ${ }^{20}$ although it has also been reported that resting cells are affected by cyclophosphamide. ${ }^{21}$ We found, in the bone marrow of cyclophosphamide-treated mice, an increase in the number of myeloid blasts, which are derived from the early blasts, already at day 4 and this increase was even greater at day 5. This rapid recovery from granulocytopenia is in agreement with the general idea that cyclophosphamide affects only the dividing precursor cells in the bone marrow. This is supported by our in vitro studies; in 7-day cultures of bonemarrow cells obtained from cyclophosphamide-treated mice the total number of cells increased from $1 \times 10^{4}$ to about $50 \times 10^{4}$ cells, while in naive mice the total number of cells increased to about $12 \times 10^{4}$ cells only. These results indicate that after cyclophosphamide treatment the remaining bonemarrow cells are triggered to proliferate faster.

G-CSF administration to cyclophosphamide-treated, noninfected mice increased mainly the number of early blasts and myeloid blasts, probably by a shortened cell-cycle time of the granulocyte precursors in the bone marrow. ${ }^{22,23}$ During infection in cyclophosphamide-treated mice that had received G-CSF, the number of early blasts in the bone marrow decreased and that of the myeloid cells did not change, but an enhanced mobilization of mature and immature granulocytes into the circulation occurred. This 10-fold increased mobilisation of mature and immature, non-dividing, granulocytes from bone marrow to the circulation during infection in G-CSF-treated mice, can be explained in part by the increased production of granulocyte precursor cells in the bone marrow induced by G-CSF, because the number of early and myeloid blasts blasts in the bone marrow of G-CSF-treated mice was about two times higher in comparison with that in the bone marrow of non-G-CSF-treated, cyclophosphamide-treated mice. However, an increase of immature circulating granulocytes after cyclophosphamide treatment, whether or not combined with G-CSF administration, has been reported recently. ${ }^{24-26}$ The mechanisms underlying the mobilization of mature and immature cells from bone marrow into the circulation are unknown as yet.

\section{ACKNOWLEDGMENTS}

This study was financially supported by the J. H. Cohen Institute for Radiopathology and Radioprotection.

\section{REFERENCES}

1. Athens J.W. (1993) Granulocytes-neutrophils. In: Wintrobe's Clinical Hematology, Leukocytes-the Phagocytic and Immunologic Systems (eds G. R. Lee, T. C. Bithel, J. Foerster, J. W. Athens \& J. N. Lukens), 9th edn, Vol 1, p. 233. Lea and Febiger, Philadelphia, London.

2. American Society of Clinical Oncology. (1994) Recommendations for the use of hematopoietic colony-stimulating factors: evidence-based, clinical practice Guidelines J Clin Oncol 12, 2471.

3. American Society of Clinical Oncology (1996) Update of recommendations for the use of hematopoietic colony-stimulating factors. Evidence-based, clinical practice guidelines. J Clin Oncol 14, 1957

4. Buisman A.M., Langermans J.A.M. \& Van Furth R. (1996) Effect of G-CSF on the course of infection with Gram-positive bacteria in mice during granulocytopenia induced by sublethal irradiation or cyclophosphamide. J Infect Dis 174, 417.

5. Leenen P.J.M., Melis M., Slieker W.A. \& Van EwiJk W. (1990) Murine macrophage precursor characterization. II. Monoclonal antibodies against precursor antigen. Eur J Immunol 20, 27.

6. Van Der Loo J.C.M., Slieker W.A.T., Kieboom D. \& PloEmaCher R.E. (1995) Identification of hematopoietic stem cell populations on the basis of their primitiveness using antibody ER-MP12. Blood 85, 952.

7. De Bruin M.F.T.R., Slieker W.A.T., Van Der Loo J.C.M., Voerman J.S.A., Van EwiJK W. \& LeEnen P.J.M. (1994) Distinct mouse bone marrow macrophage precursors identified by differential expression of ER-MP12 and ER-MP20 antigens. Eur J Immunol 24, 2279

8. de Bruin M.F.T.R., Van Vianen W., Ploemacher R.E., BakkerWoudenberg, I.A.J.M., Campbell, P.A., Van EwiJk, W. \& LeENEN, P.J.M. (1998) Bone marrow celular composition in Listeria monocytogenes infected mice detected using ER-MP12 and ER-MP20 antibodies: a flow cytometric alternative to differential counting. J Immunol Methods 217, 27.

9. Van Der loo J.C.M., Slieker W.A.T. \& Ploemacher R.E. (1995) Use of ER-MP12 as a positive marker for the isolation of murine long-term in vitro repopulating stem cells. Exp Hematol 23, 1002.

10. Chervenick P.A., Boggs D.R., Marsh J.C., Cartwright G.E. \& Wintrobe M.M. (1968) Quantitative studies of blood and bone marrow neutrophils in normal mice. J Physiol 215, 353.

11. NetA R. (1992) Radiation, effects on the immune system. In: Encyclopedia of Immunology (eds I. M. Roitt \& P. J. Delves) Vol 3, p. 1298. Academic Press, London.

12. Hoyer M. \& Nielsen O.S. (1992) Influence of dose on 
regeneration of murine hematopoietic stem cells after total body irradiation and 5-fluorouracil. Oncol 49, 166.

13. Jones T.D., Morris M.D., Young R.W. \& Kehlet R.A. (1993) A cell-kinetics model for radiation-induced myelopoiesis. Exp Hematol 21, 816 .

14. Vavrova J. \& Petyrek P. (1986) Late effects of single and repeated sublethal gamma radiations on haemopoietic stem cells in mice. Folia Biol 32, 399.

15. Down J.D., Boudewijn A., Van Os R., Thames H.D. \& Ploemacher R.E. (1995) Variations in radiation sensitivity and repair among different hematopoietic stem cell populations following fractionated irradiation. Blood 86, 122.

16. Denenkamp J. (1986) Cell kinetics and radiation biology. Int $J$ Radiat Biol 49, 357.

17. Tanikawa S., Nakao I., Tsuneoka K. \& Nara N. (1998) Effects of recombinant granulocyte colony-stimulating factor (rG-CSF) and recombinant granulocyte-macrophage colony-stimulating factor (rGM-CSF) on acute radiation hematopoietic injury in mice. Exp Hematol 17, 883.

18. Waddick K.G., Song C.W., Souza L. \& Uckun F.M. (1991) Comparative analysis of the in vivo radioprotective effects of recombinant granulocyte colony-stimulating factor (rG-CSF), recombinant granulocyte-macrophage $\mathrm{CSF}$, and their combination. Blood 11, 2364.

19. Sureda A., Valls A., Kadar E. et al. (1993) A single dose of granulocyte colony-stimulating factor modifies radiation-induced death in $\mathrm{B}_{6} \mathrm{D}_{2} \mathrm{~F}_{1}$ mice. Exp Hematol 21, 1605.

20. Ratajczak M.Z., Ratajczak J., Kuczynski W., Light B., Lusk E.J. \& GerwirTZ A.M. (1993) In vitro sensitivity of human hematopoietic progenitor cells to 4-hydroperoxycyclophosphamide. Exp Hematol 21, 1663.

21. Loo T.L. \& Freireich E.J. (1994) Cancer chemotherapeutic drugs. In: Principles of Pharmacology (eds P. L. Munson, R. A. Mueller \& G. R. Breese) p. 1478. Chapman \& Hall, New York.

22. Kabaya K., Kusaka M. \& Seki M. (1994) Effect of recombinant human granulocyte colony-stimulating factor on variations of morphologically identifiable bone marrow cells in myelosuppressed mice. In Vivo $\mathbf{8}, 1033$

23. Uchida T. \& Yamagiwa A. (1992) Kinetics of rG-CSF-induced neutrophilia in mice. Exp Hematol 20, 152.

24. Roberts A.W. \& Metcalf D. (1995) Noncycling state of peripheral blood progenitor cells mobilized by granulocyte colonystimulating factor and other cytokines. Blood 86, 1600.

25. Roberts A.W. \& Metcalf D. (1994) Granulocyte colonystimulating factor induces selective elevations of progenitor cells in the peripheral blood of mice. Exp Hematol 22, 1156.

26. Neben S., Marcus K. \& Mauch P. (1993) Mobilization of hematopoietic stem and progenitor cell subpopulations from the marrow to the blood of mice following cyclophosphamide and/or granulocyte colony-stimulating factor. Blood 81, 1960. 